\title{
Efficient Solutions of Coupled Matrix and Matrix Differential Equations
}

\author{
Zeyad Al-Zhour \\ Department of Basic Sciences and Humanities, College of Engineering, University of Dammam, \\ Dammam, KSA \\ Email: zeyad1968@yahoo.com, zalzhour@ud.edu.sa
}

Received March 7, 2012; revised April 27, 2012; accepted May 4, 2012

\begin{abstract}
In Kronecker products works, matrices are some times regarded as vectors and vectors are some times made in to matrices. To be precise about these reshaping we use the vector and diagonal extraction operators. In the present paper, the results are organized in the following ways. First, we formulate the coupled matrix linear least-squares problem and present the efficient solutions of this problem that arises in multistatic antenna array processing problem. Second, we extend the use of connection between the Hadamard (Kronecker) product and diagonal extraction (vector) operator in order to construct a computationally-efficient solution of non-homogeneous coupled matrix differential equations that useful in various applications. Finally, the analysis indicates that the Kronecker (Khatri-Rao) structure method can achieve good efficient while the Hadamard structure method achieve more efficient when the unknown matrices are diagonal.
\end{abstract}

Keywords: Matrix Products; Least-Squares Problem; Coupled Matrix and Matrix Differential Equations; Diagonal Extraction Operator

\section{Introduction}

Linear matrix and matrix differential equations show up in various fields including engineering, mathematics, physics, statistics, control, optimization, economic, linear system and linear differential system problems. For instance, the Lyapunov equations $A^{*} X+X A+Q=0$ and $X-A^{*} X A=Q$ (where $A^{*}$ is the conjugate transpose of $A$ ) are used to analyze of the stability of continuous-time and discrete-time systems, respectively [1]. The generalized Lyapunov equation:

$$
A X B^{T}+C X D^{T}=Q .
$$

(where $B^{T}$ is the transpose of $B$ ) has been used to characterize structured covariance matrices [2]. Most of the existing results, however, are connected with particular systems of such matrix and matrix differential equations.
Coupled matrix and matrix differential equations have also been widely used in stability theory of differential equations, control theory, communication systems, perturbation analysis of linear and non-linear matrix equations and other fields of pure and applied mathematics and also recently in the context of the analysis and numerical simulation of descriptor systems. For instance, the canonical system

$$
\begin{aligned}
& X^{\prime}(t)=A X(t)+B Y(t), \\
& Y^{\prime}(t)=C X(t)-A^{T} Y(t) .
\end{aligned}
$$

With the boundary conditions and $Y(b)=0$ has been used to the solution of optimal control problem with the performance index [3]. In addition, many interesting problems lead to coupled Riccati matrix differential equations [4]:

$$
\begin{aligned}
X_{1}^{\prime}(t)= & \left\{Q_{1}(t)+B_{1}(t) X_{1}(t)+X_{1}(t) A_{1}(t)+X_{1}(t) S_{11}(t) X_{1}(t)\right. \\
& \left.+X_{1}(t) S_{22}(t) X_{2}(t)+X_{2}(t) S_{22}(t) X_{1}(t)+X_{2}(t) S_{12}(t) X_{2}(t)\right\} ; \\
X_{2}^{\prime}(t)= & \left\{Q_{2}(t)+B_{2}(t) X_{2}(t)+X_{2}(t) A_{2}(t)+X_{2}(t) S_{22}(t) X_{2}(t)\right. \\
& \left.+X_{2}(t) S_{11}(t) X_{1}(t)+X_{1}(t) S_{11}(t) X_{2}(t)+X_{1}(t) S_{21}(t) X_{1}(t)\right\} . \\
X_{1}\left(t_{f}\right)= & X_{1 f}, X_{2}\left(t_{f}\right)=X_{2 f},
\end{aligned}
$$


and the general class of non-homogeneous coupled matrix differential equations:

$$
\begin{aligned}
& X_{1}^{\prime}(t)=A_{11} X_{1}(t) B_{11}+A_{12} X_{2}(t) B_{12}+\cdots+A_{1 p} X_{p}(t) B_{1 p}+U_{1}(t) \\
& X_{2}^{\prime}(t)=A_{21} X_{1}(t) B_{21}+A_{22} X_{2}(t) B_{22}+\cdots+A_{2 p} X_{p}(t) B_{2 p}+U_{2}(t) \\
& \vdots \\
& X_{p}^{\prime}(t)=A_{p 1} X_{1}(t) B_{p 1}+A_{p 2} X_{2}(t) B_{p 2}+\cdots+A_{p p} X_{p}(t) B_{p p}+U_{p}(t) .
\end{aligned}
$$

where $A_{i j}, B_{i j} \in M_{n}$ are given scalar matrices, $U_{i}(t) \in M_{n}$ is a given matrix function, $X_{i}(t) \in M_{n}$ are the unknown diagonal matrix functions to be solved and $X_{i}(0)=C_{i}$; and where $X_{i}(t)$ denotes the derivative of matrix function $X_{i}(t) .(i, j=1,2, \cdots, p)$. (where $M_{m, n}$ is the set of all $m \times n$ matrices over the complex number field $\mathbb{C}$ and when $m=n$, we write $M_{m}$ instead of $\left.M_{m, n}\right)$.

Examples of such situation are singular [5] and hybrid system control [6] and nonzero sum differential games [7]. Depending on the problem considered, different coupling terms may appear. However, in all the above mentioned cases the systems are difficult to solve.

Let us recall some concepts that will be used below. Given two matrices $A=\left[a_{i j}\right] \in M_{m, n}$ and $B=\left[b_{i j}\right] \in$ $M_{p, q}$, then the Kronecker product of $A$ and $B$ is defined by (e.g. [8-12])

$$
A \otimes B=\left[a_{i j} B\right]_{i j} \in M_{m p, n q} .
$$

While if $A \in M_{m, n}, B \in M_{p, n}$, and let $\left\{a_{i}: 1 \leq i \leq n\right\}$ and $\left\{b_{i}: 1 \leq i \leq n\right\}$ be the columns of $A$ and $B$, respectively, namely

$$
A=\left[\begin{array}{llll}
a_{1} & a_{2} & \cdots & a_{n}
\end{array}\right], \quad B=\left[\begin{array}{llll}
b_{1} & b_{2} & \cdots & b_{n}
\end{array}\right] .
$$

The columns of the Kronecker product $A \otimes B$ are $\left\{a_{i} \otimes b_{j}\right\}$ for all $i, j$ combinations in lexicographic order namely,

$$
A \otimes B=\left[a_{1} \otimes b_{1} \cdots a_{1} \otimes b_{n} \cdots a_{n} \otimes b_{1} \cdots a_{n} \otimes b_{n}\right]
$$

Thus, the Khatri-Rao product of $A$ and $B$ is defined by [13,14]:

$$
A \Theta B=\left[\begin{array}{llll}
a_{1} \otimes b_{1} & a_{2} \otimes b_{2} & \cdots & a_{n} \otimes b_{n}
\end{array}\right]
$$

consists of a subset of the columns of $A \otimes B$. Notice that $A \otimes B$ is of order $m p \times n^{2}$ and $A \Theta B$ is of order $m p \times n$. This observation can be expressed in the following form [15]:

$$
(A \otimes B) S_{n}=A \Theta B,
$$

where the selection matrix $S_{n}$ is of order $n^{2} \times n$ and

$$
S_{n}=\left[\begin{array}{lllll}
e_{1} & e_{n+2} & e_{2 n+3} & \cdots & e_{n^{2}}
\end{array}\right]
$$

and $e_{k}$ is an $n^{2} \times 1$ column vector with a unity element in the $k$-th position and zeros elsewhere $\left(1 \leq k \leq n^{2}\right)$.

Additionally, if both matrices $A=\left[a_{i j}\right]$ and
$B=\left[b_{i j}\right] \in M_{m, n}$ have the same size, then the Hadamard product of $A$ and $B$ is defined by [8-11,16]:

$$
A \circ B=\left[a_{i j} b_{i j}\right] \in M_{m, n} .
$$

This product is much simpler than Kronecker and Khatri-Rao products and it can be connected with isomorphic diagonal matrix representations that can have a certain interest in many fields of pure and applied mathematics, for example, Tauber [16] applied the Hadamard product to solving a partial differential equation coming from an air pollution problem. The Hadamard product is clearly commutative, associative, and distributive with respect to addition. It has been known that $A \circ B$ is a (principal) submatrix of $A \otimes B$ if $A$ and $B$ are (square) of the same size. This can be found in Visick [12] and even in Zhang's book [17]. Liv-Ari [13, Theorem 3.1, p. 128] gave the following new relations related to Kronecker, Khatri-Rao and Hadamard products:

$$
\begin{aligned}
& S_{n}^{T}(A \Theta B)=A \circ B ; \\
& S_{n}^{T}(A \otimes B) S_{n}=A \circ B .
\end{aligned}
$$

The Kronecker product and vector operator affirming their capability of solving some matrix and matrix differential equations. Such equations can be readily converted into the standard linear equation form by using the well-known identity (e.g. [17,18]):

$$
\operatorname{Vec}\left(A X B^{T}\right)=(B \otimes A) \operatorname{Vec} X,
$$

Where $\operatorname{Vec}($.$) denotes a vectorization by columns of$ a matrix. The need to compute the $\mathrm{e}^{A}, \cosh (A)$ and $\sinh (A)$ are due its appearance in the solutions of coupled matrix differential equations. Here

$$
\begin{gathered}
e^{A}=\sum_{k=0}^{\infty}\left(\frac{A^{k}}{k !}\right) ; \sinh (A)=\frac{\mathrm{e}^{A}-\mathrm{e}^{-A}}{2} ; \\
\cosh (A)=\frac{\mathrm{e}^{A}+\mathrm{e}^{-A}}{2} .
\end{gathered}
$$

For any matrix $A \in M_{m}$, the spectral representation of $\mathrm{e}^{A}$ and $\mathrm{e}^{A t}$ assures that $[9,18]$ :

$$
\mathrm{e}^{A}=\sum_{i=0}^{n} x_{i} y_{i}^{T} \mathrm{e}^{\lambda_{i}} ; \mathrm{e}^{A t}=\sum_{i=0}^{n} x_{i} y_{i}^{T} \mathrm{e}^{\lambda_{i} t},
$$

where $\left\{\lambda_{1}, \cdots, \lambda_{n}\right\}$ and $\left\{x_{1}, \cdots, x_{n}\right\}$ are the eigenvalues and the corresponding eigenvectors of $A$, and $\left\{y_{1}, \cdots, y_{n}\right\}$ 
is the eigenvectors of matrix $A^{T}$.

Finally, for any matrices $A, B, C, D \in M_{n}$, we shall make a frequent use the following properties of the Kronecker product (e.g. $[9,18-20])$ which are used to establish our results.

1) $(A \otimes B)(C \otimes D)=A C \otimes B D ;(A \otimes B)^{*}=A^{*} \otimes B^{*}$

2) $e^{\left(A \otimes I_{n}+I_{n}+B\right)}=e^{A} \otimes e^{B} ; \quad e^{\left(A \otimes I_{n}\right)}=e^{A} \otimes I_{n}$;

$$
e^{\left(I_{n} \otimes B\right)}=I_{n} \otimes e^{A}
$$

3) $\sinh \left(A \otimes I_{n}\right)=(\sinh A) \otimes I_{n}$;

$$
\sinh \left(I_{n} \otimes B\right)=I_{n} \otimes(\sinh B)
$$

4) $\cosh \left(A \otimes I_{n}\right)=(\cosh A) \otimes I_{n}$;

$$
\cosh \left(I_{n} \otimes B\right)=I_{n} \otimes(\cosh B) .
$$

In this paper, we present the efficient solution of coupled matrix linear least-squares problem and extend the use of diagonal extraction (vector) operator in order to construct a computationally-efficient solution of nonhomogeneous coupled matrix linear differential equations.

\section{Coupled Matrix Linear Least-Squares Problem}

The multistatic antenna array processing problem can be written in matrix notation as [13]

$$
Q=A X B^{T} ; X=\operatorname{diag}\left(\tau_{i} ; 1 \leq i \leq n\right) .
$$

where $A \in M_{m, n}, \quad B \in M_{p, n}$ and $Q \in M_{m, p}$ are given (complex valued) matrices; and where the unknown matrix $X \in M_{n}$ is diagonal. We also assume that $n<m p$, so that we suggest using a least-squares approach, viz.,

$$
\min _{X}\left\|Q-A X B^{T}\right\|_{F}^{2},
$$

where $\|A\|_{F}$ is called Frobenius norm of $A$. Using the identity in Equation (13) we can transform (21) into the vector LSP form:

$$
\min _{X}\|\operatorname{Vec} Q-(B \otimes A) \operatorname{Vec} X\|_{F}^{2} .
$$

which has the well-known solution:

$$
\operatorname{Vec} X=\left((B \otimes A)^{*}(B \otimes A)\right)^{-1}(B \otimes A)^{*} \operatorname{Vec} Q,
$$

provided $(B \otimes A)^{*}(B \otimes A)$ is invertible.

Applying the direct vector transformation in Equation (13) to $Q-A X B^{T}$ results in a highly inefficient leastsquare problem, because $V e c X$ is very sparse. Liv-Ari [13] described an alternative approach based on:

$$
\operatorname{Vec}\left(A X B^{T}\right)=(B \Theta A) \operatorname{Vecd}(X), X \text { is diagonal }
$$

which involves the so-called Khatri-Rao product $\Theta$, as well as the diagonal extraction operator vecd $(X)$ :

$$
\operatorname{Vecd}(X)=\left(\begin{array}{llll}
x_{11} & x_{22} & \cdots & x_{n n}
\end{array}\right)^{T}
$$

which forms a column vector consisting of the diagonal elements of the $n \times n$ square matrix $X$, instead of the much longer column vector $V e c X$. In addition, if $Y$ is any matrix of order $m \times p$, then

$$
\operatorname{Vecd}\left(A^{T} Y B\right)=(B \Theta A)^{T} \operatorname{Vec} Y .
$$

As we have observed earlier, when the unknown matrix $X$ is diagonal, solving for $\operatorname{Vec} X$ is highly inefficient, since most of the elements of $X$ vanish. Instead Liv-Ari [13] used the more compact vectorization identity to rewrite matrix LSP (21) in the vector form:

$$
\min _{X}\|\operatorname{Vec} Q-(B \Theta A) \operatorname{Vecd}(X)\|_{F}^{2} .
$$

Notice that $\operatorname{Vecd}(X)$ consists of only the nontrivial (i.e., diagonal) elements of the matrix $X$. The explicit solution of (27) is

$$
\operatorname{Vecd}(X)=\left((B \Theta A)^{*}(B \Theta A)\right)^{-1}(B \Theta A)^{*} \operatorname{Vec} Q .
$$

provided $(B \Theta A)^{*}(B \Theta A)$ is invertible.

It turns out that this expression can also be implemented using Hadamard product, resulting in a significant reduction in computational cost, as implied the following result [13]:

$$
(A \Theta B)^{*}(A \Theta B)=\left(A^{*} A\right) \circ\left(B^{*} B\right),
$$

where $A \in M_{m, n}$ and $B \in M_{p, n}$.

When $n<\min \{m, p\}$, we observe that the left-hand side expression in Equation (29) requires

$m p n+m p n(n+1) / 2$ multiplications, while forming the equivalent right-hand side expression requires only $(m+p+1) n(n+1) / 2$ multiplications. Thus the latter offers significant computational savings, especially when $m p \gg m+p+1$.

Now, using (26) we can rewrite (28) in the more compact form:

$$
\operatorname{Vecd}(X)=\left(\left(B^{*} B\right) \circ\left(A^{*} A\right)\right)^{-1} \operatorname{Vecd}\left\{A^{*} Q \operatorname{conj}(B)\right\} .
$$

This expression which requires $O\left(n^{3}\right)+O\left([m+p] n^{2}\right)$ (multiply and add) operations is much more efficient than (28), which requires $O\left(n^{3}\right)+O\left(m p n^{2}\right)$ operations. It means that the computational advantage of using the Hadamard product expression is particularly evident when $n<\min \{m, p\}$, which implies that

$m p \gg m+p \gg n$. In order to be able to use (30) we must ascertain that the matrix $\left(B^{*} B\right) \circ\left(A^{*} A\right)$ is invertible. This will hold, for instance, when both $A$ and $B$ have full 
column rank.

As for the diagonal extraction operator vecd(.), we observe that for any square $n \times n$ matrix $Y=\left[y_{i j}\right]$,

$$
\operatorname{Vecd}(Y)=S_{n}^{T} \operatorname{Vec} Y \text {. }
$$

If $Y$ is diagonal, then we also have

$$
\operatorname{Vec} Y=S_{n} \operatorname{Vecd}(Y), Y \text { is diagonal. }
$$

Moreover, the columns of the $n^{2} \times n$ selection matrix $S_{n}$ are mutually orthonormal, viz.,

$$
S_{n}^{T} S_{n}=I_{n} .
$$

Using (32) and (11), we get the fundamental relation between the Hadamard product and diagonal extraction operator vecd (.) which is given by

$$
\operatorname{Vecd}\left(A X B^{T}\right)=(B \circ A) \operatorname{Vecd}(X), X \text { is diagonal }
$$

where $A, B$ and $X$ is $n \times n$ diagonal matrix.

Now we will discuss the efficient and more efficient least-squares solutions of coupled matrix linear equations:

$$
A X C^{T}+B Y C^{T}=E, \quad B X C^{T}+A Y C^{T}=F
$$

where $A, B \in M_{m, n} C \in M_{p, n}, E, F \in M_{m, p}$ are given scalar matrices and $X, Y \in M_{n}$ are unknown matrices to be solved. We also assume that $n<m p$, so that the coupled matrix linear Equations (35) is over-determined, which suggests using a least squares approach. We con- sider the coupled matrix linear least-squares problem (CLSP):

$$
\min _{X, Y}\left\|\left[\begin{array}{l}
E \\
F
\end{array}\right]-\left[\begin{array}{l}
A X C^{T}+B Y C^{T} \\
B X C^{T}+A Y C^{T}
\end{array}\right]\right\|_{F}^{2}
$$

The solution procedure presented here may be considered as a continuation of the method proposed to solve least-squares problem in (21).

Using the identity (13) we can transform (36) into the vector CLSP form [10]:

$$
\min _{X, Y}\left\|\left[\begin{array}{l}
V e c E \\
V e c F
\end{array}\right]-\left[\begin{array}{cc}
C \otimes A & C \otimes B \\
C \otimes B & C \otimes A
\end{array}\right]\left[\begin{array}{l}
V e c X \\
V e c Y
\end{array}\right]\right\|_{F}^{2}
$$

which has the following solution

$$
\begin{aligned}
& {\left[\begin{array}{l}
V e c X \\
V e c Y
\end{array}\right]=\left(\left[\begin{array}{cc}
C \otimes A & C \otimes B \\
C \otimes B & C \otimes A
\end{array}\right]^{*}\left[\begin{array}{ll}
C \otimes A & C \otimes B \\
C \otimes B & C \otimes A
\end{array}\right]\right)^{-1}} \\
& \times\left[\begin{array}{ll}
C \otimes A & C \otimes B \\
C \otimes B & C \otimes A
\end{array}\right]^{*}\left[\begin{array}{c}
V e c E \\
V e c F
\end{array}\right] .
\end{aligned}
$$

One can easily show that

$$
\left[\begin{array}{ll}
C \otimes A & C \otimes B \\
C \otimes B & C \otimes A
\end{array}\right]=U\left[\begin{array}{cc}
C \otimes(A+B) & 0 \\
0 & C \otimes(A-B)
\end{array}\right] U^{T}
$$

where $U=\frac{1}{\sqrt{2}}\left[\begin{array}{cc}I & -I \\ I & I\end{array}\right]$ is a unitary matrix. So

$$
\begin{aligned}
{\left[\begin{array}{cc}
C \otimes A & C \otimes B \\
C \otimes B & C \otimes A
\end{array}\right]^{*}\left[\begin{array}{cc}
C \otimes A & C \otimes B \\
C \otimes B & C \otimes A
\end{array}\right] } & =U\left[\begin{array}{cc}
(C \otimes(A+B))^{*} & 0 \\
0 & (C \otimes(A-B))^{*}
\end{array}\right] U^{T} \times U\left[\begin{array}{cc}
C \otimes(A+B) & 0 \\
0 & C \otimes(A-B)
\end{array}\right] U^{T} \\
& =U\left[\begin{array}{cc}
(C \otimes(A+B))^{*}(C \otimes(A+B)) & 0 \\
0 & (C \otimes(A-B))^{*}(C \otimes(A-B))
\end{array}\right] U^{T}
\end{aligned}
$$

Suppose that $H=C \otimes(A+B)$ and $W=C \otimes(A-B)$, we then have

$$
\begin{aligned}
& \left(\left[\begin{array}{cc}
C \otimes A & C \otimes B \\
C \otimes B & C \otimes A
\end{array}\right]^{*}\left[\begin{array}{cc}
C \otimes A & C \otimes B \\
C \otimes B & C \otimes A
\end{array}\right]\right)^{-1}=U\left[\begin{array}{cc}
\left((C \otimes(A+B))^{*}(C \otimes(A+B))\right)^{-1} & 0 \\
0 & \left((C \otimes(A-B))^{*}(C \otimes(A-B))\right)^{-1}
\end{array}\right] U^{T} \\
& =\frac{1}{2}\left[\begin{array}{cc}
I & -I \\
I & I
\end{array}\right]\left[\begin{array}{cc}
\left(H^{*} H\right)^{-1} & 0 \\
0 & \left(W^{*} W\right)^{-1}
\end{array}\right]\left[\begin{array}{cc}
I & I \\
-I & I
\end{array}\right]=\frac{1}{2}\left[\begin{array}{cc}
\left(H^{*} H\right)^{-1}+\left(W^{*} W\right)^{-1} & \left(H^{*} H\right)^{-1}-\left(W^{*} W\right)^{-1} \\
\left(H^{*} H\right)^{-1}-\left(W^{*} W\right)^{-1} & \left(H^{*} H\right)^{-1}+\left(W^{*} W\right)^{-1}
\end{array}\right] .
\end{aligned}
$$

Now the least—squares solutions (38) can be rewrite into the form:

$$
\left[\begin{array}{c}
\operatorname{Vec} X \\
\operatorname{Vec} Y
\end{array}\right]=\frac{1}{2}\left[\begin{array}{ll}
\left(H^{*} H\right)^{-1}+\left(W^{*} W\right)^{-1} & \left(H^{*} H\right)^{-1}-\left(W^{*} W\right)^{-1} \\
\left(H^{*} H\right)^{-1}-\left(W^{*} W\right)^{-1} & \left(H^{*} H\right)^{-1}+\left(W^{*} W\right)^{-1}
\end{array}\right] \times\left[\begin{array}{ll}
(C \otimes A)^{*} & (C \otimes B)^{*} \\
(C \otimes B)^{*} & (C \otimes A)^{*}
\end{array}\right]\left[\begin{array}{c}
\operatorname{Vec} E \\
\operatorname{Vec} F
\end{array}\right] .
$$


This gives

$$
\begin{aligned}
\operatorname{Vec} X= & \frac{1}{2} \times\left\{\left(\left(H^{*} H\right)^{-1}+\left(W^{*} W\right)^{-1}\right)(C \otimes A)^{*}+\left(\left(H^{*} H\right)^{-1}-\left(W^{*} W\right)^{-1}\right)(C \otimes B)^{*}\right\} \times V e c E \\
& +\frac{1}{2} \times\left\{\left(\left(H^{*} H\right)^{-1}+\left(W^{*} W\right)^{-1}\right)(C \otimes B)^{*}+\left(\left(H^{*} H\right)^{-1}-\left(W^{*} W\right)^{-1}\right)(C \otimes A)^{*}\right\} \times V e c F \\
V e c Y= & \frac{1}{2}\left\{\left(\left(H^{*} H\right)^{-1}-\left(W^{*} W\right)^{-1}\right)(C \otimes A)^{*}+\left(\left(H^{*} H\right)^{-1}+\left(W^{*} W\right)^{-1}\right)(C \otimes B)^{*}\right\} \times V e c E \\
& +\frac{1}{2}\left\{\left(\left(H^{*} H\right)^{-1}-\left(W^{*} W\right)^{-1}\right)(C \otimes B)^{*}+\left(\left(H^{*} H\right)^{-1}+\left(W^{*} W\right)^{-1}\right)(C \otimes A)^{*}\right\} \times V e c F
\end{aligned}
$$

where $H=C \otimes(A+B)$ and $W=C \otimes(A-B)$.

In order to be able to use (38) and (43) we must ascertain that the matrix:

$$
\left[\begin{array}{ll}
C \otimes A & C \otimes B \\
C \otimes B & C \otimes A
\end{array}\right]^{*}\left[\begin{array}{ll}
C \otimes A & C \otimes B \\
C \otimes B & C \otimes A
\end{array}\right]
$$

is invertible if and only one

$$
H^{*} H=(C \otimes(A+B))^{*}(C \otimes(A+B))
$$

and

$$
W^{*} W=(C \otimes(A-B))^{*}(C \otimes(A-B))
$$

are invertible matrices.
As we observed, when the unknown matrices $X$ and $Y \in M_{n}$ are diagonal, solving for $\operatorname{Vec} X$ and $V e c Y$ are highly inefficient, since most of the elements of $X$ and $Y$ vanish. Instead we can use the more compact vectorization identity (24) to rewrite the coupled matrix linear least-squares problem (37) in the reduced-order vector form:

$$
\min _{X, Y}\left\|\left[\begin{array}{l}
V e c E \\
\operatorname{Vec} F
\end{array}\right]-\left[\begin{array}{cc}
C \Theta A & C \Theta B \\
C \Theta B & C \Theta A
\end{array}\right]\left[\begin{array}{l}
\operatorname{Vecd}\{X\} \\
\operatorname{Vecd}\{Y\}
\end{array}\right]\right\|_{F}^{2} .
$$

Notice that $\operatorname{Vecd}\{X\}$ and $\operatorname{Vecd}\{Y\}$ consists of only the nontrivial (i.e., diagonal) elements of matrices $X$ and $Y$. The explicit efficient solution of (44) is

$$
\begin{aligned}
\operatorname{Vecd}\{X\}= & \frac{1}{2}\left\{\left(\left(R^{*} R\right)^{-1}+\left(S^{*} S\right)^{-1}\right)(C \Theta A)^{*}+\left(\left(R^{*} R\right)^{-1}-\left(S^{*} S\right)^{-1}\right)(C \Theta B)^{*}\right\} \times \operatorname{Vec} E \\
& +\frac{1}{2}\left\{\left(\left(R^{*} R\right)^{-1}+\left(S^{*} S\right)^{-1}\right)(C \Theta B)^{*}+\left(\left(R^{*} R\right)^{-1}-\left(S^{*} S\right)^{-1}\right)(C \Theta A)^{*}\right\} \times \operatorname{Vec} F \\
\operatorname{Vecd}\{Y\}= & \frac{1}{2}\left\{\left(\left(R^{*} R\right)^{-1}-\left(S^{*} S\right)^{-1}\right)(C \Theta A)^{*}+\left(\left(R^{*} R\right)^{-1}+\left(S^{*} S\right)^{-1}\right)(C \Theta B)^{*}\right\} \times V e c E \\
& +\frac{1}{2}\left\{\left(\left(R^{*} R\right)^{-1}-\left(S^{*} S\right)^{-1}\right)(C \Theta B)^{*}+\left(\left(R^{*} R\right)^{-1}+\left(S^{*} S\right)^{-1}\right)(C \Theta A)^{*}\right\} \times V e c F
\end{aligned}
$$

where $R=C \Theta(A+B)$ and $S=C \Theta(A-B)$.

In order to be able to use (45), we must ascertain that the matrix

$$
R^{*} R=(C \Theta(A+B))^{*}(C \Theta(A+B))
$$

and

$$
S^{*} S=(C \Theta(A-B))^{*}(C \Theta(A-B))
$$

are invertible matrices.

It turns out that the expression (45) can also be implemented using Hadamard product by the same technique in the expression (30). Note that the least squares solutions in term of Hadamard product is more efficient than (45) and (43).

\section{Non-Homogeneous Matrix Differential Equations}

The solution procedure presented here may be considered as a continuation of the method proposed to solve the homogenous coupled matrix differential equations in [18]. We will use our knowledge of the solution of the of simplest homogeneous matrix differential equation:

$$
X^{\prime}(t)=A X(t), \quad X(0)=C
$$

where $A \in M_{m}, C \in M_{m, n}$ are given scalar matrices, and $X(t) \in M_{m, n}$ is the unknown matrix function to be solved. In fact the unique solution of (46) is given by:

$$
X(t)=\mathrm{e}^{A t} C
$$

Theorem 3.1 Let $A \in M_{m}, C \in M_{m, n}$ are given scalar matrices, $U(t) \in M_{m, n}$ is a given matrix function and $X(t) \in M_{m, n}$ is the unknown matrix. Then the general solution of the non-homogeneous matrix differential equation:

$$
X^{\prime}(t)=A X(t)+U(t), \quad X(0)=C
$$


is given by

$$
X(t)=\mathrm{e}^{A t} C+\mathrm{e}^{A t} * U(t) .
$$

Where $\mathrm{e}^{A t} * U(t)=\int_{0}^{t} \mathrm{e}^{A(t-s)} U(s) \mathrm{d} s$ is well-defined, which involves the convolution product of two matrices $\mathrm{e}^{A t}$ and $U(t)$.

Proof: Suppose that $X_{p}(t)=\mathrm{e}^{A t} G(t)$ is the particular solution of (48). The product rule of differentiation gives

$$
X_{p}(t)=\mathrm{e}^{A t} G^{\prime}(t)+A \mathrm{e}^{A t} G(t) .
$$

Substituting these in (48) we obtain

$$
\mathrm{e}^{A t} G^{\prime}(t)+A \mathrm{e}^{A t} G(t)=A \mathrm{e}^{A t} G(t)+U(t)
$$

Thus

$$
\mathrm{e}^{A t} G^{\prime}(t)=U(t)
$$

Multiplying both sides of (50) by $\mathrm{e}^{-A t}=\left(\mathrm{e}^{A t}\right)^{-1}$ gives

$$
G^{\prime}(t)=\mathrm{e}^{-A t} U(t)
$$

Integrating both sides of (51) between 0 and $t$ gives

$$
G(t)=\int_{0}^{t} \mathrm{e}^{-A s} U(s) \cdot \mathrm{d} s
$$

Hence, by assumption, we conclude that the particular solution of equation (48) is

$$
X_{p}(t)=\mathrm{e}^{A t} G(t)=\int_{0}^{t} \mathrm{e}^{A(t-s)} U(s) \mathrm{d} s=\mathrm{e}^{A t} * U(t) .
$$

Now from (47) and (53) we get (49).

Theorem 3.2 Let $A=\left[a_{i j}\right], B=\left[b_{i j}\right], C \in M_{n}$ are given scalar matrices, $U(t) \in M_{n}$ is a given matrix function and $X(t) \in M_{n}$ is unknown diagonal matrix function. Then the general solution of non-homogeneous matrix differential equation

$$
X^{\prime}(t)=A X(t)+X(t) B+U(t), X(0)=C
$$

is given by

$$
\operatorname{Vecd}\{X(t)\}=\operatorname{diag}\left(\mathrm{e}^{\left(a_{11}+b_{11}\right) t}, \cdots, \mathrm{e}^{\left(a_{n n}+b_{n n}\right) t}\right) \operatorname{Vecd}\{C\}+\operatorname{diag}\left(\mathrm{e}^{\left(a_{11}+b_{11}\right) t}, \cdots, \mathrm{e}^{\left(a_{n n}+b_{n n}\right) t}\right) * \operatorname{Vecd}\{U(t)\} .
$$

Proof: Using the identity (34) we can transform (54) into the vector form:

$$
\begin{aligned}
\operatorname{Vecd}\left\{X^{\prime}(t)\right\} & =\left(I_{n} \circ A+B^{T} \circ I_{n}\right) \operatorname{Vecd}\{X(t)\}+\operatorname{Vecd}\{U(t)\}=\left\{\left(A+B^{T}\right) \circ I_{n}\right\} \operatorname{Vecd}\{X(t)\}+\operatorname{Vecd}\{U(t)\} \\
& =\operatorname{diag}\left(a_{11}+b_{11}, \cdots, a_{n n}+b_{n n}\right) \operatorname{Vecd}\{X(t)\}+\operatorname{Vecd}\{U(t)\} .
\end{aligned}
$$

Now, applying (49), then the unique solution of (56) is

$$
\begin{aligned}
\operatorname{Vecd}\{X(t)\} & =\mathrm{e}^{\operatorname{diag}\left(a_{11}+b_{11}, \cdots, a_{n n}+b_{n n}\right) t} \operatorname{Vecd}\{C\}+\mathrm{e}^{\operatorname{diag}\left(a_{11}+b_{11}, \cdots, a_{n n}+b_{n n}\right) t} * \operatorname{Vecd}\{U(t)\} \\
& =\operatorname{diag}\left(\mathrm{e}^{\left(a_{11}+b_{11}\right) t}, \cdots, \mathrm{e}^{\left(a_{n n}+b_{n n}\right) t}\right) \operatorname{Vecd}\{C\}+\operatorname{diag}\left(\mathrm{e}^{\left(a_{11}+b_{11}\right) t}, \cdots, \mathrm{e}^{\left(a_{n n}+b_{n n}\right) t}\right) * \operatorname{Vecd}\{U(t)\} .
\end{aligned}
$$

If we put $U(t)=0$ in Theorem 3.2 we obtain the following result.

Corollary 3.3 Let $A, B, C \in M_{n}$ are given scalar matrices. Then general solution of the homogeneous matrix differential equation:

$$
\begin{aligned}
& X^{\prime}(t)=A X(t)+X(t) B, X(0)=C, \\
& X(t) \text { is diagonal. }
\end{aligned}
$$

is given by

$$
\operatorname{Vecd}\{X(t)\}=\operatorname{diag}\left(\mathrm{e}^{\left(a_{11}+b_{11}\right) t}, \cdots, \mathrm{e}^{\left(a_{n n}+b_{n n}\right) t}\right) \operatorname{Vecd}\{C\}
$$

Now we will discuss the general class of non-homogeneous coupled matrix differential equations which defined in (4): By using the Vecd (.) -notation of (4), we have

$$
\left[\begin{array}{c}
\operatorname{Vecd}\left\{X_{1}^{\prime}(t)\right\} \\
\operatorname{Vecd}\left\{X_{2}^{\prime}(t)\right\} \\
\vdots \\
\operatorname{Vecd}\left\{X_{p}^{\prime}(t)\right\}
\end{array}\right]=\left[\begin{array}{cccc}
B_{11}^{T} \circ A_{11} & B_{12}^{T} \circ A_{12} & \cdots & B_{1 p}^{T} \circ A_{1 p} \\
B_{21}^{T} \circ A_{21} & B_{22}^{T} \circ A_{22} & \cdots & B_{2 p}^{T} \circ A_{2 p} \\
\vdots & \vdots & \vdots & \vdots \\
B_{p 1}^{T} \circ A_{p 1} & B_{p 2}^{T} \circ A_{p 2} & \cdots & B_{p p}^{T} \circ A_{p p}
\end{array}\right] \times\left[\begin{array}{c}
\operatorname{Vecd}\left\{X_{1}(t)\right\} \\
\operatorname{Vecd}\left\{X_{2}(t)\right\} \\
\vdots \\
\operatorname{Vecd}\left\{X_{p}(t)\right\}
\end{array}\right]+\left[\begin{array}{c}
\operatorname{Vecd}\left\{U_{1}(t)\right\} \\
\operatorname{Vecd}\left\{U_{2}(t)\right\} \\
\vdots \\
\operatorname{Vecd}\left\{U_{p}(t)\right\}
\end{array}\right]
$$

Let 


$$
\begin{gathered}
x^{\prime}(t)=\left[\begin{array}{c}
\operatorname{Vecd}\left\{X_{1}^{\prime}(t)\right\} \\
\operatorname{Vecd}\left\{X_{2}^{\prime}(t)\right\} \\
\vdots \\
\operatorname{Vecd}\left\{X_{p}^{\prime}(t)\right\}
\end{array}\right], x(t)=\left[\begin{array}{c}
\operatorname{Vecd}\left\{X_{1}(t)\right\} \\
\operatorname{Vecd}\left\{X_{2}(t)\right\} \\
\vdots \\
\operatorname{Vecd}\left\{X_{p}(t)\right\}
\end{array}\right], c=\left[\begin{array}{c}
\operatorname{Vecd}\left\{C_{1}\right\} \\
\operatorname{Vecd}\left\{C_{2}\right\} \\
\vdots \\
\operatorname{Vecd}\left\{C_{p}\right\}
\end{array}\right], u(t)=\left[\begin{array}{c}
\operatorname{Vecd}\left\{U_{1}(t)\right\} \\
\operatorname{Vecd}\left\{U_{2}(t)\right\} \\
\vdots \\
\operatorname{Vecd}\left\{U_{p}(t)\right\}
\end{array}\right] \\
H=\left[\begin{array}{cccc}
B_{11}^{T} \circ A_{11} & B_{12}^{T} \circ A_{12} & \cdots & B_{1 p}^{T} \circ A_{1 p} \\
B_{21}^{T} \circ A_{21} & B_{22}^{T} \circ A_{22} & \cdots & B_{2 p}^{T} \circ A_{2 p} \\
\vdots & \vdots & \vdots & \vdots \\
B_{p 1}^{T} \circ A_{p 1} & B_{p 2}^{T} \circ A_{p 2} & \cdots & B_{p p}^{T} \circ A_{p p}
\end{array}\right] .
\end{gathered}
$$

Now (59) can be written as

$$
x^{\prime}(t)=H x(t)+u(t), x(0)=c
$$

and the general solution is given by:

$$
x(t)=\mathrm{e}^{H t} c+\mathrm{e}^{H t} * u(t) .
$$

Note that there is many special cases can be considered from the above general class coupled matrix differential equations; now we will discuss some important special cases in the next results.

Theorem 3.4 Let $A, B, C, D, E, F \in M_{n}$ are given scalar matrices such that

$$
\begin{gathered}
\left(D^{T} \circ C\right)\left(B^{T} \circ A\right)=\left(B^{T} \circ A\right)=\left(D^{T} \circ C\right) ; \\
U(t), V(t) \in M_{n}
\end{gathered}
$$

are given matrix functions and $X(t), Y(t) \in M_{n}$ are the unknown diagonal matrices. Then the general solution of non-homogeneous coupled matrix differential equations:

$$
\begin{aligned}
X^{\prime}(t)= & A X(t) B+C Y(t) D+U(t), \\
Y^{\prime}(t)= & C X(t) D+A Y(t) B+V(t), \\
& X(0)=E, Y(0)=F
\end{aligned}
$$

is given by

$$
\begin{aligned}
\operatorname{Vecd}\{X(t)\}= & \mathrm{e}^{\left(B^{T} \circ A\right) t}\left\{\left[\cosh \left(D^{T} \circ C\right) t\right] \operatorname{Vecd}\{E\}+\left[\sinh \left(D^{T} \circ C\right) t\right] \operatorname{Vecd}\{F\}\right\} \\
& +\mathrm{e}^{\left(B^{T} \circ A\right) t} \times\left\{\left[\cosh \left(D^{T} \circ C\right) t\right] * \operatorname{Vecd}\{U(t)\}+\left[\sinh \left(D^{T} \circ C\right) t\right] * \operatorname{Vecd}\{V(t)\}\right\} \operatorname{Vecd}\{Y(t)\} \\
\operatorname{Vecd}\{Y(t)\}= & \mathrm{e}^{\left(B^{T} \circ A\right) t}\left\{\left[\sinh \left(D^{T} \circ C\right) t\right] \operatorname{Vecd}\{E\}+\left[\cosh \left(D^{T} \circ C\right) t\right] \operatorname{Vecd}\{F\}\right\} \\
& +\mathrm{e}^{\left(B^{T} \circ A\right) t}\left\{\left[\sinh \left(D^{T} \circ C\right) t\right] * \operatorname{Vecd}\{U(t)\}+\left[\cosh \left(D^{T} \circ C\right) t\right] * \operatorname{Vecd}\{V(t)\}\right\}
\end{aligned}
$$

Proof: Using the identity (34) we can transform (62) into the vector form:

$$
\left[\begin{array}{l}
\operatorname{Vecd}\left\{X^{\prime}(t)\right\} \\
\operatorname{Vecd}\left\{Y^{\prime}(t)\right\}
\end{array}\right]=\left[\begin{array}{cc}
B^{T} \circ A & D^{T} \circ C \\
D^{T} \circ C & B^{T} \circ A
\end{array}\right]\left[\begin{array}{l}
\operatorname{Vecd}\{X(t)\} \\
\operatorname{Vecd}\{Y(t)\}
\end{array}\right]+\left[\begin{array}{l}
\operatorname{Vecd}\{U(t)\} \\
\operatorname{Vecd}\{V(t)\}
\end{array}\right]
$$

From (61), this system has the following solution:

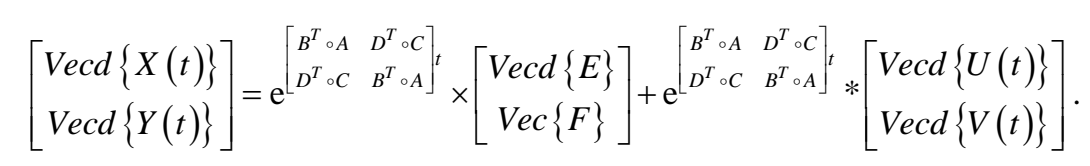

Now we will deal with

$$
\mathrm{e}^{\left[\begin{array}{cc}
B^{T} \circ{ }^{\circ} \circ & D^{T}{ }^{\circ} \circ \\
D^{T} C & B^{T} \circ A
\end{array}\right]}
$$$$
\begin{aligned}
& {\left[\begin{array}{cc}
B^{T} \circ A & 0 \\
0 & B^{T} \circ A
\end{array}\right]\left[\begin{array}{cc}
0 & D^{T} \circ C \\
D^{T} \circ C & 0
\end{array}\right]} \\
& =\left[\begin{array}{cc}
0 & D^{T} \circ C \\
D^{T} \circ C & 0
\end{array}\right]\left[\begin{array}{cc}
B^{T} \circ A & 0 \\
0 & B^{T} \circ A
\end{array}\right] .
\end{aligned}
$$

Since $\left(D^{T} \circ C\right)\left(B^{T} \circ A\right)=\left(B^{T} \circ A\right)=\left(D^{T} \circ C\right)$, then we have

Then 


$$
\begin{aligned}
\mathrm{e}^{\left[\begin{array}{cc}
B^{T} \circ A & D^{T} \circ C \\
D^{T} \circ C & B^{T} \circ A
\end{array}\right]} & =\mathrm{e}^{\left[\begin{array}{cc}
B^{T} \circ A & 0 \\
0 & B^{T} \circ A
\end{array}\right]+\left[\begin{array}{cc}
0 & D^{T} \circ C \\
D^{T} \circ C & 0
\end{array}\right]} \\
& =\mathrm{e}^{\left[\begin{array}{cc}
B^{T} \circ A & 0 \\
0 & B^{T} \circ A
\end{array}\right]} \cdot e^{\left[\begin{array}{cc}
0 & D^{T} \circ C \\
D^{T} \circ C & 0
\end{array}\right]} .
\end{aligned}
$$

$$
\begin{aligned}
& \mathrm{e}^{\left[\begin{array}{cc}
B^{T} \circ A & 0 \\
0 & B^{T} \circ A
\end{array}\right]}=\left[\begin{array}{cc}
\mathrm{e}^{\left(B^{T} \circ A\right)} & 0 \\
0 & \mathrm{e}^{\left(B^{T} \circ A\right)}
\end{array}\right]
\end{aligned}
$$

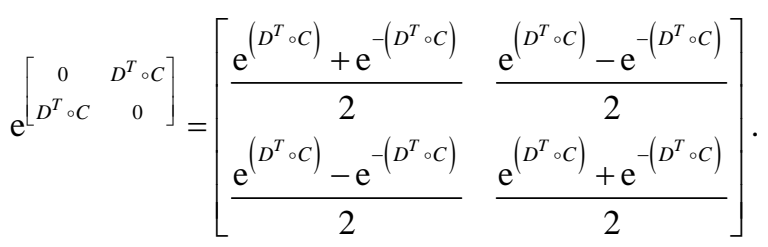

$$
\begin{aligned}
& \mathrm{e}^{\left[\begin{array}{ll}
B^{T} \circ A & D^{T} \circ C \\
D^{T} \circ C & B^{T} \circ A
\end{array}\right]_{t}} \times\left[\begin{array}{l}
\operatorname{Vecd}\{E\} \\
\operatorname{Vecd}\{F\}
\end{array}\right]=\left[\begin{array}{ll}
\mathrm{e}^{\left(B^{T} \circ A\right) t} \cosh \left(D^{T} \circ C\right) t & \mathrm{e}^{\left(B^{T} \circ A\right) t} \sinh \left(D^{T} \circ C\right) t \\
\mathrm{e}^{\left(B^{T} \otimes A\right) t} \sinh \left(D^{T} \circ C\right) t & \mathrm{e}^{\left(B^{T} \circ A\right) t} \cosh \left(D^{T} \circ C\right) t
\end{array}\right] \times\left[\begin{array}{l}
\operatorname{Vecd}\{E\} \\
\operatorname{Vecd}\{F\}
\end{array}\right]
\end{aligned}
$$

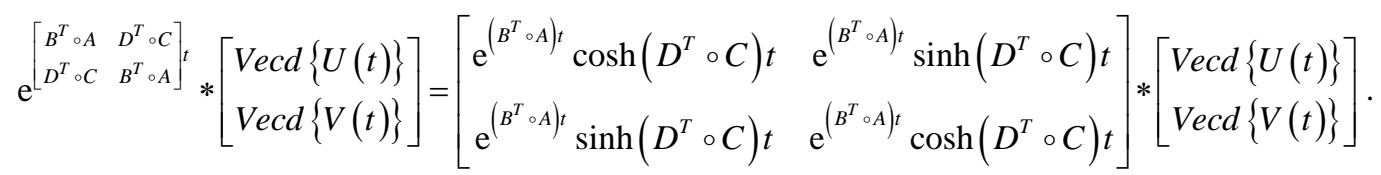

$$
\begin{aligned}
& \mathrm{e}^{\left[\begin{array}{cc}
B^{T} \circ A & D^{T} \circ C \\
D^{T} \circ C & B^{T} \circ A
\end{array}\right]}=\left[\begin{array}{cc}
\mathrm{e}^{\left(B^{T} \circ A\right)} & 0 \\
0 & \mathrm{e}^{\left(B^{T} \circ A\right)}
\end{array}\right] \\
& \times\left[\begin{array}{ll}
\frac{\mathrm{e}^{\left(D^{T} \circ C\right)}+e^{-\left(D^{T} \circ C\right)}}{2} & \frac{\mathrm{e}^{\left(D^{T} \circ C\right)}-\mathrm{e}^{-\left(D^{T} \circ C\right)}}{2} \\
\frac{\mathrm{e}^{\left(D^{T} \circ C\right)}-\mathrm{e}^{-\left(D^{T} \circ C\right)}}{2} & \frac{\mathrm{e}^{\left(D^{T} \circ C\right)}+\mathrm{e}^{-\left(D^{T} \circ C\right)}}{2}
\end{array}\right] \\
& =\left[\begin{array}{ll}
\mathrm{e}^{\left(B^{T} \circ A\right)} \cosh \left(D^{T} \circ C\right) & \mathrm{e}^{\left(B^{T} \circ A\right)} \sinh \left(D^{T} \circ C\right) \\
\mathrm{e}^{\left(B^{T} \circ A\right)} \sinh \left(D^{T} \circ C\right) & \mathrm{e}^{\left(B^{T} \circ A\right)} \cosh \left(D^{T} \circ C\right)
\end{array}\right]
\end{aligned}
$$

Now substitute (68) and (69) in (65), we get (63).

If we put $U(t)=V(t)=0$ in Theorem 3.4 we obtain the following result.

Corollary 3.5 Let $A, B, C, D, E, F \in M_{n}$ are given scalar matrices such that

$$
\left(D^{T} \circ C\right)\left(B^{T} \circ A\right)=\left(B^{T} \circ A\right)=\left(D^{T} \circ C\right),
$$

and $X(t), Y(t) \in M_{n}$ are the unknown diagonal ma-

$$
\begin{aligned}
& \operatorname{Vecd}\{X(t)\}=\mathrm{e}^{\left(B^{T} \circ A\right) t}\left\{\left[\cosh \left(D^{T} \circ C\right) t\right] \operatorname{Vecd}\{E\}+\left[\sinh \left(D^{T} \circ C\right) t\right] \operatorname{Vecd}\{F\}\right\} ; \\
& \operatorname{Vecd}\{Y(t)\}=\mathrm{e}^{\left(B^{T} \circ A\right) t}\left\{\left[\sinh \left(D^{T} \circ C\right) t\right] \operatorname{Vecd}\{E\}+\left[\cosh \left(D^{T} \circ C\right) t\right] \operatorname{Vecd}\{F\}\right\} .
\end{aligned}
$$

Corollary 3.6 Let $B=\left[b_{i j}\right], \quad D=\left[d_{i j}\right], E, \quad F \in M_{n}$ are given scalar matrices and $X(t), Y(t) \in M_{n}$ are the unknown diagonal matrices. Then the general solution of homogeneous coupled matrix differential equations: trices. Then the general solution of homogeneous coupled matrix differential equations:

$$
\begin{gathered}
X^{\prime}(t)=A X(t) B+C Y(t) D, \\
Y^{\prime}(t)=C X(t) D+A Y(t) B, \\
X(0)=E, Y(0)=F
\end{gathered}
$$

is given by

$$
\begin{aligned}
& \operatorname{Vecd}\{X(t)\}=\left\{\operatorname{diag}\left(\mathrm{e}^{b_{11} t} \cosh d_{11} t, \cdots, \mathrm{e}^{b_{n n} t} \cosh d_{n n} t\right)\right\} \operatorname{Vecd}\{E\}+\left\{\operatorname{diag}\left(\mathrm{e}^{b_{11} t} \sinh d_{11} t, \ldots, \mathrm{e}^{b_{n n} t} \sinh d_{n n} t\right)\right\} \operatorname{Vecd}\{F\} \\
& \operatorname{Vecd}\{Y(t)\}=\left\{\operatorname{diag}\left(\mathrm{e}^{b_{11} t} \sinh d_{11} t, \cdots, \mathrm{e}^{b_{n n} t} \sinh d_{n n} t\right)\right\} \operatorname{Vecd}\{E\}+\left\{\operatorname{diag}\left(\mathrm{e}^{b_{11} t} \cosh d_{11} t, \ldots, \mathrm{e}^{b_{n n} t} \cosh d_{n n} t\right)\right\} \operatorname{Vecd}\{F\}
\end{aligned}
$$

Proof: For any matrix $A=\left[a_{i j}\right] \in M_{n}$, it is easily to show that

$$
\cosh \left(A^{T} \circ I\right) t=\operatorname{diag}\left(\cosh \left(a_{11} t\right), \cosh \left(a_{22} t\right), \cdots, \cosh \left(a_{n n} t\right)\right) ;
$$




$$
\sinh \left(A^{T} \circ I\right) t=\operatorname{diag}\left(\sinh \left(a_{11} t\right), \sinh \left(a_{22} t\right), \cdots, \sinh \left(a_{n n} t\right)\right) .
$$

Now put $A=C=I_{n}$ in Corollary 3.5 we have

$$
\begin{aligned}
\operatorname{Vecd}\{X(t)\}= & \mathrm{e}^{\left(B^{T} \otimes I_{n}\right) t}\left\{\left[\cosh \left(D^{T} \circ I_{n}\right) t\right] \operatorname{Vecd}\{E\}+\left[\sinh \left(D^{T} \circ I_{n}\right) t\right] \operatorname{Vecd}\{F\}\right\} \\
= & \left\{\operatorname{diag}\left(\mathrm{e}^{b_{11} t}, \cdots, \mathrm{e}^{b_{n n} t}\right) \cdot \operatorname{diag}\left(\cosh d_{11} t, \cdots, \cosh d_{n n} t\right)\right\} \operatorname{Vecd}\{E\} \\
& +\left\{\operatorname{diag}\left(\mathrm{e}^{b_{11} t}, \cdots, \mathrm{e}^{b_{n n} t}\right) \cdot \operatorname{diag}\left(\sinh d_{11} t, \cdots, \sinh d_{n n} t\right)\right\} \operatorname{Vecd}\{F\} \\
= & \left\{\operatorname{diag}\left(\mathrm{e}^{b_{11} t} \cosh d_{11} t, \cdots, \mathrm{e}^{b_{n n} t} \cosh d_{n n} t\right)\right\} \operatorname{Vecd}\{E\}+\left\{\operatorname{diag}\left(\mathrm{e}^{b_{11} t} \sinh d_{11} t, \ldots, \mathrm{e}^{b_{n n} t} \sinh d_{n n} t\right)\right\} \operatorname{Vecd}\{F\}
\end{aligned}
$$

Similarly,

$$
\operatorname{Vecd}\{Y(t)\}=\left\{\operatorname{diag}\left(\mathrm{e}^{b_{11} t} \sinh d_{11} t, \cdots, \mathrm{e}^{b_{n n} t} \sinh d_{n n} t\right)\right\} \operatorname{Vecd}\{E\}+\left\{\operatorname{diag}\left(\mathrm{e}^{b_{11} t} \cosh d_{11} t, \cdots, \mathrm{e}^{b_{n n} t} \cosh d_{n n} t\right)\right\} \operatorname{Vecd}\{F\} .
$$

While if we applying the fundamental relation between $\operatorname{Vec}($.$) and Kronecker product defined in (13) and using$ the same technique in the proof of Theorem 3.4 we obtain (for any matrix $X \in M_{n}$ ) the following result.

Theorem 3.7 Let $A, B, C, D, E, F \in M_{n}$ are given scalar matrices such that $A C=C A, B D=D B, U(t)$, $V(t) \in M_{n}$ are given matrix functions and $X(t)$, $Y(t) \in M_{n}$ are the unknown matrices. Then the general solution of non-homogeneous coupled matrix differential equations:

$$
\begin{gathered}
X^{\prime}(t)=A X(t) B+C Y(t) D+U(t) \\
Y^{\prime}(t)=C X(t) D+A Y(t) B+V(t) \\
X(0)=E, Y(0)=F
\end{gathered}
$$

is given by

$$
\begin{aligned}
\operatorname{Vec} X(t)= & \mathrm{e}^{\left(B^{T} \otimes A\right) t}\left\{\left[\cosh \left(D^{T} \otimes C\right) t\right] \operatorname{Vec} E+\left[\sinh \left(D^{T} \otimes C\right) t\right] \operatorname{Vec} F\right\} \\
& +\mathrm{e}^{\left(B^{T} \otimes A\right) t}\left\{\left[\cosh \left(D^{T} \otimes C\right) t\right] * \operatorname{Vec} U(t)+\left[\sinh \left(D^{T} \otimes C\right) t\right] * \operatorname{Vec} V(t)\right\} \\
\operatorname{Vec} Y(t)= & \mathrm{e}^{\left(B^{T} \otimes A\right) t}\left\{\left[\sinh \left(D^{T} \otimes C\right) t\right] \operatorname{Vec} E+\left[\cosh \left(D^{T} \otimes C\right) t\right] \operatorname{Vec} F\right\} \\
& +\mathrm{e}^{\left(B^{T} \otimes A\right) t}\left\{\left[\sinh \left(D^{T} \otimes C\right) t\right] * \operatorname{Vec} U(t)+\left[\cosh \left(D^{T} \otimes C\right) t\right] * \operatorname{VecV}(t)\right\}
\end{aligned}
$$

If we put $U(t)=V(t)=0$ and $A=C=I_{n}$ in Theorem 3.7 and using properties (16)-(19) we obtain the following results.

Corollary 3.8 Let $B, D, E, F \in M_{n}$ are given scalar matrices such that $B D=D B$ and $X(t), Y(t) \in M_{n}$ are the unknown matrices. Then the general solution of homogeneous coupled matrix differential equations:

$$
\begin{gathered}
X^{\prime}(t)=X(t) B+Y(t) D, \\
Y^{\prime}(t)=X(t) D+Y(t) B, \\
X(0)=E, Y(0)=F
\end{gathered}
$$

is given by

$$
\begin{aligned}
& X(t)=\{E \cosh (D t)+F \sinh (D t)\} \mathrm{e}^{B t}, \\
& Y(t)=\{E \sinh (D t)+F \cosh (D t)\} \mathrm{e}^{B t} .
\end{aligned}
$$

Corollary 3.9 Let $A, C, E, F \in M_{n}$ are given scalar matrices such that $A C=C A$ and $X(t), Y(t) \in M_{n}$ are the unknown matrices.Then the general solution of homogeneous coupled matrix differential equations:

$$
\begin{gathered}
X^{\prime}(t)=A X(t)+C Y(t), \\
Y^{\prime}(t)=C X(t)+A Y(t), \\
X(0)=E, Y(0)=F
\end{gathered}
$$

is given by

$$
\begin{aligned}
& X(t)=\mathrm{e}^{A t}\{\cosh (C t) \cdot E+\sinh (C t) \cdot F\}, \\
& Y(t)=\mathrm{e}^{A t}\{\sinh (C t) \cdot E+\cosh (C t) \cdot F\} .
\end{aligned}
$$

\section{Concluding Remarks}

We have studied an explicit characterization of the mappings

$$
A \otimes B \Rightarrow A \Theta B \Rightarrow A \circ B
$$

in terms of the selection matrix $S_{n}$ as in (11) and (12). 
We have also observed that the same matrix relates the two operators $\operatorname{Vec}($.$) and Vecd (.) as in (31) and (32).$ We used the fundamental relation between the Hadamard (Kronecker) product and diagonal extraction (vector) operator in (34) and (13) to derive our main results in Section 2 and 3 and, subsequently, to construct a computationally-efficient solution of coupled matrix least-squares problem and non-homogeneous coupled matrix differential equations. In fact, the Kronecker (Hadamard) product and operator $\operatorname{Vec}($.$) (Vecd (.)) affirming their capabil-$ ity of solving matrix and matrix differential equations fast (more fast when the unknown matrices are diagonal). To demonstrate the usefulness of applying some properties of the Kronecker products, suppose we have to solve, for example, the following system:

$$
B X A^{T}=C,
$$

where $A, B \in M_{n}$ are given scalar matrices and $X \in M_{n}$ is unknown matrix to be solved. Then it is not hard by using the $\operatorname{Vec}($.$) -notation to establish the following$ equivalence:

$$
(A \otimes B) \operatorname{Vec} X=\operatorname{Vec} C,
$$

and thus also by using the $\operatorname{Vec}($.$) -notation product to$ establish the following equivalence:

$$
(A \circ B) \operatorname{Vecd}(X)=\operatorname{Vecd}(C), X \text { is diagonal. }
$$

If we ignore the Kronecker (Hadamard) product structure, then we need to solve the following both matrix equations:

$$
\text { - } B Y=C
$$

Here, $Y$ can be obtained in $O\left(n^{3}\right)$ arithmetic operations (flops) by using $L U$ factorization of matrix $B$ (Forward Substitution).

- $X A^{T}=Y$

Here $X$ can be obtained also in $O\left(n^{3}\right)$ operations (flops) by using $L U$ factorization of matrix A (Back Sub-

stitution).

Now without exploiting the Kronecker product structure, an $n^{2} \times n^{2}$ system defined in (82) would normally (by Gaussian elimination) require $O\left(n^{6}\right)$ operations to solve. But when we use Kronecker product structure: $(A \otimes B) \operatorname{Vec} X=\operatorname{Vec} C$, the calculations shows that $\operatorname{Vec} X$ can be obtained only in $O\left(n^{3}\right)$ operations by using $L U$ factorization of matrices $A$ and $B$ [20, pp. 87]. We can say that the system of the form: $(A \otimes B) \operatorname{Vec} X=\operatorname{Vec} C$ can be solved fast and the Kronecker structure also a voids the formation of $n^{2} \times n^{2}$ matrices, only the smaller lower and upper triangular matrices $L_{A}, L_{B}, U_{A}, U_{B}$ are needed. While if $X$ is $n \times n$ diagonal matrix and use the Hadamard product structure: $(A \circ B) \operatorname{Vecd}(X)=\operatorname{Vecd}(C)$, the calculations shows that $\operatorname{Vecd}(X)$ can be obtained only in $O(n)$ operations by using $L U$ factorization of $A \circ B$.

We can say that the system of the form: $(A \circ B) \operatorname{Vecd}(X)=\operatorname{Vecd}(C)$ can be solved more fast than Kronecker structure, only the very smaller lower and upper triangular matrices $L_{A \circ B}$ and $U_{A \circ B}$ are needed. For example, consider $A, B$ are $3 \times 3$ matrices and $C$ is $9 \times 1$ vector. To demonstrate the usefulness of applying Kronecker product and $\operatorname{Vec}($.$) -notation, we$ return to the system problem $(A \otimes B) \operatorname{Vec} X=V e c C$. If $A \otimes B$ is non-singular and regarding with $L U$ factorizetions of $A=L_{A} U_{A}$ and $B=L_{B} U_{B}$, then a solution of system exists and can be written as:

$$
\left(U_{A} \otimes U_{B}\right) \operatorname{Vec} X=z,\left(L_{A} \otimes L_{B}\right) z=\operatorname{Vec} C .
$$

First, the lower triangular system $\left(L_{A} \otimes L_{B}\right) z=V e c C$ can be solved by forward substitution as the following:

$$
\left(\left[\begin{array}{ccc}
a_{11} & 0 & 0 \\
a_{21} & a_{22} & 0 \\
a_{31} & a_{32} & a_{33}
\end{array}\right] \otimes\left[\begin{array}{ccc}
b_{11} & 0 & 0 \\
b_{21} & b_{22} & 0 \\
b_{31} & b_{32} & b_{33}
\end{array}\right]\right)\left[\begin{array}{c}
z_{1} \\
z_{2} \\
\vdots \\
z_{9}
\end{array}\right]=\left[\begin{array}{c}
c_{1} \\
c_{2} \\
\vdots \\
c_{9}
\end{array}\right]
$$

i.e.,

$$
L_{A} \otimes L_{B}=\left[\begin{array}{ccccccccc}
a_{11} b_{11} & 0 & 0 & 0 & 0 & 0 & 0 & 0 & 0 \\
a_{11} b_{21} & a_{11} b_{22} & 0 & 0 & 0 & 0 & 0 & 0 & 0 \\
a_{11} b_{31} & a_{11} b_{32} & a_{11} b_{33} & 0 & 0 & 0 & 0 & 0 & 0 \\
a_{21} b_{11} & 0 & 0 & a_{22} b_{11} & 0 & 0 & 0 & 0 & 0 \\
a_{21} b_{21} & a_{21} b_{22} & 0 & a_{22} b_{21} & a_{22} b_{22} & 0 & 0 & 0 & 0 \\
a_{21} b_{31} & a_{21} b_{32} & a_{21} b_{33} & a_{22} b_{31} & a_{22} b_{32} & a_{22} b_{33} & 0 & 0 & 0 \\
a_{31} b_{11} & 0 & 0 & a_{32} b_{11} & 0 & 0 & a_{33} b_{11} & 0 & 0 \\
a_{31} b_{21} & a_{31} b_{22} & 0 & a_{32} b_{21} & a_{32} b_{22} & 0 & a_{33} b_{21} & a_{33} b_{22} & 0 \\
a_{31} b_{31} & a_{31} b_{32} & a_{31} b_{33} & a_{32} b_{31} & a_{32} b_{32} & a_{32} b_{33} & a_{33} b_{31} & a_{33} b_{32} & a_{33} b_{33}
\end{array}\right]
$$


which can be solved in $O\left(n^{2}\right)=O(9)$ operations. The first three equations are:

- $a_{11} b_{11} z_{1}=c_{1} \Rightarrow z_{1}=\frac{c_{1}}{a_{11} b_{11}}$.

- $a_{11} b_{21} z_{1}+a_{11} b_{22} z_{2}=c_{2} \Rightarrow z_{2}=\frac{b_{11} c_{2}-b_{21} c_{1}}{a_{11} b_{11} b_{22}}$.

- $a_{11} b_{31} z_{1}+a_{11} b_{32} z_{2}+a_{11} b_{33} z_{3}=c_{3}$

$$
\Rightarrow z_{3}=\frac{b_{11} b_{22} c_{3}-b_{11} b_{32} c_{2}-b_{22} b_{31} c_{1}+b_{32} b_{21} c_{1}}{a_{11} b_{11} b_{22} b_{33}}
$$

Now the next three equations are:

- $\boldsymbol{a}_{21} \boldsymbol{b}_{11} \mathrm{z}_{1}+a_{22} b_{11} z_{4}=c_{4}$.

- $\boldsymbol{a}_{21} \boldsymbol{b}_{21} \mathbf{z}_{1}+\boldsymbol{a}_{21} \boldsymbol{b}_{12} z_{2}+a_{22} b_{21} z_{4}+a_{22} b_{22} z_{5}=c_{5}$.

- $a_{21} b_{31} z_{1}+a_{21} b_{32} z_{2}+a_{21} b_{33} z_{3}$

$$
+a_{22} b_{31} z_{4}+a_{22} b_{32} z_{5}+a_{22} b_{33} z_{6}=c_{6} .
$$

The first boldface expression $\boldsymbol{a}_{21} \boldsymbol{b}_{11} \mathbf{z}_{\mathbf{1}}$ in (91) can be computed as $\frac{a_{21} c_{1}}{a_{11}}$. The second boldface expression $\boldsymbol{a}_{21} \boldsymbol{b}_{21} \mathbf{z}_{1}+\boldsymbol{a}_{21} \boldsymbol{b}_{12} \mathbf{z}_{2}$ in (92) can be also computed as $\frac{a_{21} c_{2}}{a_{11}}$. While the third boldface expression $\boldsymbol{a}_{21} \boldsymbol{b}_{31} \mathbf{z}_{1}+\boldsymbol{a}_{21} \boldsymbol{b}_{32} \mathbf{z}_{2}+\boldsymbol{a}_{21} \boldsymbol{b}_{33} \mathbf{z}_{3}$ in (93) can be also computed as $\frac{a_{21} c_{3}}{a_{11}}$.

We use the previous expressions for obtaining $z_{1}, z_{2}$ and $z_{3}$ in the first set of equations to simplify the second set of three equations. The simplified second set of equations becomes

$$
\begin{gathered}
a_{22} b_{11} z_{4}=c_{4}-\frac{a_{21} c_{1}}{a_{11}} . \\
a_{22} b_{21} z_{4}+a_{22} b_{22} z_{5}=c_{5}-\frac{a_{21} c_{2}}{a_{11}} .
\end{gathered}
$$

$$
a_{22} b_{31} z_{4}+a_{22} b_{32} z_{5}+a_{22} b_{33} z_{6}=c_{6}-\frac{a_{21} c_{3}}{a_{11}} .
$$

Solving the second set of equations takes $O(n)$ operations and the forward solve step takes $O\left(n^{2}\right)$ operations, so obtaining $z_{4}, z_{5}$ and $z_{6}$ takes $O\left(n^{2}\right)$ time. This simplification and using the work from the previous solution step continuous so that solving each of $n$-sets of $n$-equations takes $O\left(n^{2}\right)$ time, resulting in an overall solution time of $O\left(n^{2}\right)$. Exploiting the Kronecker structure reduce the usual, expected $O\left(n^{4}\right)$ time to solve $\left(L_{A} \otimes L_{B}\right) z=V e c C$ to $O\left(n^{2}\right)$.

One final note regarding the exploitation of the Kronecker structure of the system remains. Suppose the matrices $A$ and $B$ are different sizes. Then, the time required to solve the system $(A \otimes B) \operatorname{Vec} X=\operatorname{Vec} C$ is $O\left(n_{A} n_{B}^{2}\right)$, where $n_{A}$ is the size of $A$ and $n_{B}$ is the size of $B$. In our work, the modeler has some choice for the size of the $A$ and $B$ matrices. Thus, a wise choice would make $n_{B}$ small, reducing the effect of the $n_{B}^{2}$ term in the $O\left(n_{A} n_{B}^{2}\right)$ computation time.

While when $X$ is $n \times n$ diagonal matrix and applying $\operatorname{Vecd}($.$) -notation, we return to the system problem:$ $(A \circ B) \operatorname{Vecd}(X)=\operatorname{Vecd}(C)$. If $A \circ B$ is non-singular matrix and regarding with $L U$ factorizations of $A \circ B=$ $L_{A \circ B} U_{A \circ B}$, then a solution of system exists and can be written as:

$$
U_{A \circ B} \operatorname{Vecd}(X)=y, L_{A \circ B} y=\operatorname{Vecd}(C) .
$$

First, the lower triangular system $L_{A \circ B} y=\operatorname{Vecd}(C)$ can be solved by forward substitution as the following:

$$
\left[\begin{array}{ccc}
a_{11} b_{11} & 0 & 0 \\
a_{21} b_{21} & a_{22} b_{22} & 0 \\
a_{31} b_{31} & a_{32} b_{32} & a_{33} b_{33}
\end{array}\right]\left[\begin{array}{l}
y_{1} \\
y_{2} \\
y_{3}
\end{array}\right]=\left[\begin{array}{c}
c_{11} \\
c_{22} \\
c_{33}
\end{array}\right],
$$

which can be solved in $O(n)=O(3)$ operations as follows:

- $a_{11} b_{11} y_{1}=c_{11} \Rightarrow y_{1}=\frac{c_{11}}{a_{11} b_{11}}$.

- $a_{21} b_{21} y_{1}+a_{22} b_{22} y_{2}=c_{22} \Rightarrow y_{2}=\frac{a_{11} b_{11} c_{22}-a_{21} b_{21} c_{11}}{a_{11} b_{11} a_{22} b_{22}}$.

- $a_{31} b_{31} y_{1}+a_{32} b_{32} y_{2}+a_{33} b_{33} y_{3}=c_{33} \Rightarrow y_{3}=\frac{a_{11} b_{11} a_{22} b_{22} c_{33}-\left[a_{31} b_{31} a_{22} b_{22}-a_{32} b_{32} a_{21} b_{21}\right] c_{11}-a_{32} b_{32} a_{11} b_{11} c_{22}}{a_{11} b_{11} a_{22} b_{22} a_{33} b_{33}}$.

\section{Conclusion}

The solution of coupled matrix linear least-squares problems and coupled matrix differential equations is studied and some important special cases are discussed. The analysis indicates that solving for $\operatorname{Vec}($.$) is efficient$ and solving for $\operatorname{Vecd}($.$) is more efficient when the un-$ known matrices are diagonal. Although the algorithms are presented for non-homogeneous coupled matrix and matrix linear differential equations, the idea adopted can be easily extended to study coupled matrix nonlinear differential equations, e.g., the coupled matrix Riccati 
differential equations.

\section{Acknowledgements}

The author expresses his sincere thanks to referee (s) for careful reading of the manuscript and several helpful suggestions. The author also gratefully acknowledges that this research was partially supported by Deanship of Scientific Research/University of Dammam/Kingdom of Saudi Arabia/under the Grant No. 2012152.

\section{REFERENCES}

[1] T. Kailath, "Linear Systems," Prentice-Hall, Englewood Cliffs, 1980.

[2] T. Kailath and A. H. Sayed, "Displacement Structure: Theory and Applications,” SIAM Review, Vol. 37, No. 3, 1995, pp. 297-386. doi:10.1137/1037082

[3] G. Mouroutsos and P. D. Sparis, "Taylor Series Approach to System Identification, Analysis and Optimal Control,” Journal of the Franklin Institute, Vol. 319, No. 3, pp. 359-371. doi:10.1016/0016-0032(85)90056-0

[4] L. Jódar and H. Abou-Kandil, "Kronecker Products and Coupled Matrix Riccati Differential Systems," Linear Algebra and its Applications, Vol. 121, 1989, pp. 39-51. doi:10.1016/0024-3795(89)90690-3

[5] S. Campbell, "Singular Systems of Differential Equations II,” Pitman, London, 1982.

[6] M. Mariton, "Les Systèmes Linéaires á Sauts Markoviens,” Thèse d’Etat, Université Paris-Sud, 1986.

[7] J. B. Cruz and C. I. Chen, "Series Nash Solution of TwoPerson Nonzero Sum Linear Differential Games,” Journal of Optimization Theory and Applications, Vol. 7, No. 4, 1971, pp. 240-257. doi:10.1007/BF00928706

[8] Z. Al-Zhour and A. Kilicman, "Matrix Equalities and Inequalities Involving Khatri-Rao and Tracy-Singh Sums," Journal of Inequalities in Pure \& Applied Mathematics, Vol. 7, No. 1, 2006, pp. 496-513.

[9] A. Graham, "Kronecker Products and Matrix Calculus with Applications,” Ellis Horwood Ltd., New York, 1981.
[10] A. Kilicman and Z. Al-Zhour, "Vector Least-Squares Solutions of Coupled Singular Matrix Equations," Journal of Computational and Applied Mathematics, Vol. 206, No. 2, 2007, pp. 1051-1069. doi:10.1016/j.cam.2006.09.009

[11] W.-H. Steeb, "Matrix Calculus and Kronecker Product with Applications and $\mathrm{C}_{++}$Programs,” World Scientific Publishing Co. Pte. Ltd., Singapore, 1997.

[12] G. Visick, “A Quantitative Version of the Observation that the Hadamard Product Is A Principle Submatrix of the Kronecker Product," Linear Algebra and its Applications, Vol. 304, No. 1-3, 2000, pp. 45-68. doi:10.1016/S0024-3795(99)00187-1

[13] H. Lev-Ari, "Efficient Solution of Linear Matrix Equations with Application to Multistatic Antenna Array Processing," Communications in Information \& Systems, Vol. 5, No. 1, 2005, pp. 123-130.

[14] C. R. Rao and M. B. Rao, "Matrix Algebra and Its Applications to Statistics and Econometrics,” World Scientific Publishing Co. Pte. Ltd., Singapore, 1998.

[15] F. Ding and T. Chen, "Iterative Least-Squares Solutions of Coupled Sylvester Matrix Equations,” Systems \& Control Letters, Vol. 54, No. 2, 2005, pp. 95-107. doi:10.1016/j.sysconle.2004.06.008

[16] S. Tauber, "An Applications of the Hadamard Product to Air Pollution," Applied Mathematics and Computation, Vol. 4, No. 2, 1978, pp. 167-176. doi:10.1016/0096-3003(78)90020-6

[17] F. Zhang, Matrix Theory: Basic Results and Techniques, Springer-Verlag, New York, 1999.

[18] A. Kilicman and Z. Al-Zhour, "The General Common Exact Solutions of Coupled Linear Matrix and Matrix Differential Equations," Journal of Computational Analysis and Applications, Vol. 1, No. 1, 2005, pp. 15-29.

[19] J. R. Magnus and H. Neudecker, "Matrix Differential Calculus with Applications in Statistics and Econometrics,” John Wiley and Sons Ltd., New York, 1999.

[20] G. F. Van Loan, "The Ubiquitous Kronecker Product," Journal of Computational and Applied Mathematics, Vol. 123, No. 1-2, 2000, pp. 85-100. doi:10.1016/S0377-0427(00)00393-9 